\title{
LES and RANS for Turbulent Flow over Arrays of Wall- Mounted Obstacles
}

\author{
Zhengtong Xie and Ian P Castro (i.castro@soton.ac.uk)* \\ School of Engineering Sciences, University of Southampton, Highfield, \\ Southampton SO17 1BJ
}

\begin{abstract}
Large-eddy simulation (LES) has been applied to calculate the turbulent flow over staggered wall-mounted cubes and staggered random arrays of obstacles with area density $25 \%$, at Reynolds numbers between $5 \times 10^{3}$ and $5 \times 10^{6}$, based on the free stream velocity and the obstacle height. $R e=5 \times 10^{3}$ data were intensively validated against direct numerical simulation (DNS) results at the same $R e$ and experimental data obtained in a boundary layer developing over an identical roughness and at a rather higher $R e$. The results collectively confirm that Reynolds number dependency is very weak, principally because the surface drag is predominantly form drag and the turbulence production process is at scales comparable to the roughness element sizes. LES is thus able to simulate turbulent flow over the urbanlike obstacles at high $R e$ with grids that would be far too coarse for adequate computation of corresponding smooth-wall flows. Comparison between LES and steady Reynolds-averaged Navier-Stokes (RANS) results are included, emphasising that the latter are inadequate, especially within the canopy region.
\end{abstract}

Keywords: group of obstacles, bluff body, turbulent flow, Reynolds number dependency

\section{Introduction}

A number of major observations of flow and dispersion in urban area have been completed recently and more are planned. In numerical modelling for street-scale problems, Britter \& Hanna (2003) point out that computational studies typically produce reasonable qualitative results but the performance, when compared with laboratory or field experiments, is little better than that of simple operational models. Large-eddy simulation (LES) (Sagaut, 2001) is a promising tool for computing unsteady 3-dimensional flows at high Reynolds number or with complex geometry. An LES resolves only the large-scale fluid motions and models the subgrid-scale (SGS) motions through filtering the Navier-Stokes equations. When unsteady Reynolds-averaged NavierStokes (RANS) methods are used, it is implicitly assumed that there is a fair degree of scale separation between the large time scale of the unsteady flow features and the time scale of the genuine turbulence (Castro, 2003). However, in reality it is hard to find an evident time

\footnotetext{
* This e-mail address is available for all problems and questions.
}

(C) 2006 Kluwer Academic Publishers. Printed in the Netherlands. 
scale gap for many turbulent flows. And, of course, RANS generally eliminates most of the genuinely turbulent fluctuation information.

Flow over groups of cubes mounted on a wall provides an excellent test case for validation for LES. The groups of cubes represent either simple buildings or roughness elements. Furthermore, understanding of such flows is also directly beneficial to the understanding of building aerodynamics, urban meteorology and atmospheric boundary layer meteorology (Britter \& Hanna, 2003; Stoesser et al., 2003). Dittrich et al. (1996) conducted an experiment in a tilting flume to investigate flow over staggered cubes or spheres, or aligned spheres. Cheng \& Castro (2002) performed comprehensive measurements over staggered or aligned cubes in a thick wind tunnel boundary layer at Reynolds numbers ( $R e$, based on cube height and free-stream velocity) between 5000 and 13000, and Coceal et al. (2006) conducted a direct numerical simulation (DNS) of turbulent channel flow with one wall comprising similar arrays of cubes at $R e=5000$ (based on the maximum - domain height - velocity and the height of the cubes). The latter's results are in good agreement with the former's measurements in the near-surface layer despite the mismatch in outer layer thickness. Hanna et al. (2002) used a finite element flow solver (FEFLO) with LES to simulate the mean flow and turbulence fluctuations within and above staggered or aligned cubes. Their results are compared with measurements obtained from a hydraulic water flume. Kanda et al. (2004) performed an LES (LES-CITY) to compute the flow over a square array of cubes. Their results are also in good agreement with laboratory experiments. Nevertheless, further studies applying LES to 3-D flow over obstacle arrays are still needed (Kanda et al., 2004). For instance, the issue of the Reynolds number dependency, or of the minimum resolution required for LES applied to flows over a real urban area, need to be further addressed.

Our main interest here is on the urban environment, so we are mostly concerned with the flow within and above the cube-canopy, up to a height at least equal to the top of the roughness sub-layer (the region in which the flow is spatially inhomogeneous, which typically has a depth of a few element heights at most, see Cheng \& Castro, 2002). Incompressible flow over a staggered array of cubes with an area coverage of $25 \%$ at a Reynolds numbers of 5000 based on maximum mean velocity and cube height has been intensively investigated. Higher Reynolds number cases $\left(5 \times 10^{4}\right.$ and $\left.5 \times 10^{6}\right)$ were also simulated for this test case. In addition, a further test case having a more complex geometry and also studied experimentally by Cheng \& Castro was simulated. The geometries are shown in Figures 1 and in the more complex case (Figure 1b, c) consisted of a collection of $80 \mathrm{~mm} \times 80 \mathrm{~mm}$ 'repeating' 


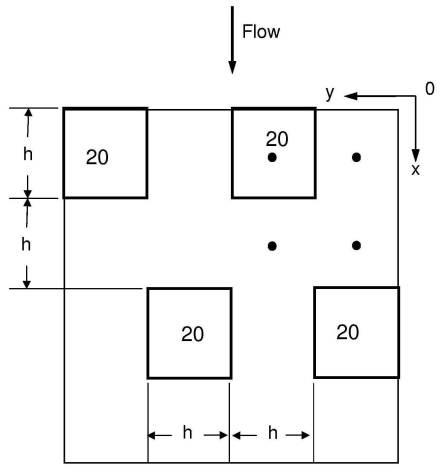

(a)

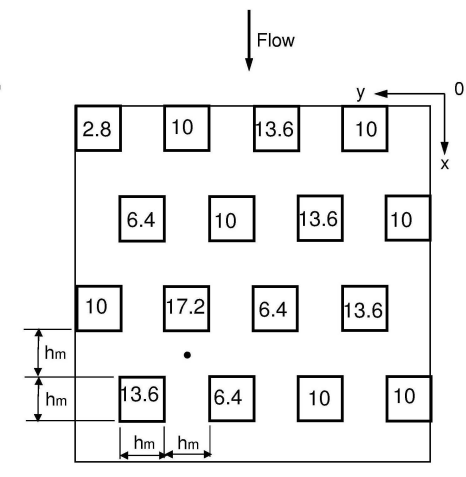

(b)

(c)

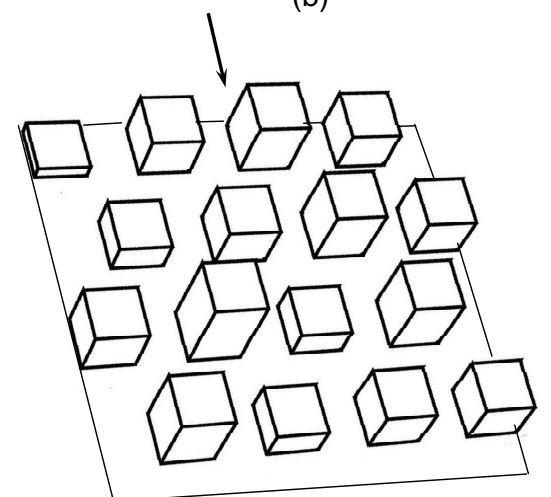

Figure 1. (a): schematic plan view of the domain for staggered cube arrays (case designated C20S); (b): schematic plan view of one unit of the domain for staggered random arrays, which consists of 2 (streamwise) $\times 2$ (lateral) units (designated RM10S). Dimensions in mm, with element heights indicated; (c): perspective view of one repeating unit of RM10S.

units, each containing sixteen 10-mm-square elements placed in a regular staggered pattern and having five different heights chosen from a normal distribution with a mean and standard deviation of $h_{m}=10 \mathrm{~mm}$ and $3 \mathrm{~mm}$ respectively. For this case, the Reynolds number based on the maximum stream velocity and the mean height of the obstacles was approximately 6000 .

As the eventual objective is to simulate such flows at much higher Reynolds number, we resorted to large-eddy simulation but not direct numerical simulation. Furthermore, comparison of LES with three Reynolds-averaged Navier-Stokes (RANS) models, namely the standard $k-\epsilon$ model (SKE), a modified $k-\epsilon$ model (MKE) and the 
Reynolds stress model (RSM), was conducted, illustrating the limitations of RANS models for canopy flows.

In Section 2 the governing equations, the subgrid-scale model(SGS), the three RANS models and the corresponding wall models are given. Section 3 presents further details and in Section 4, firstly, the grid resolution of LES is validated by comparing with Castro et al. (2005)'s measurements and Coceal et al. (2006)'s DNS data. Secondly, simulation of flows at $\operatorname{Re}=5 \times 10^{4}$ and $\mathrm{Re}=5 \times 10^{6}$ are presented and discussed and, thirdly, flows in a much larger domain consisting of 64 random obstacles are described. Lastly, a comparison of results from three RANS models with the LES data is given. Section 5 presents some final discussion and emphasises the major conclusions.

\section{Governing Equations}

\subsection{LARGE-EDDY SIMULATION}

We solve the filtered Navier-Stokes equations,

$$
\begin{aligned}
\frac{\partial u_{i}}{\partial x_{i}} & =0 \\
\frac{\partial u_{i}}{\partial t}+\frac{\partial u_{i} u_{j}}{\partial x_{j}} & =-\frac{1}{\rho}\left(\frac{\partial p}{\partial x_{i}}+\delta_{i 1} \frac{\partial<P>}{\partial x_{1}}\right)+\frac{\partial}{\partial x_{j}}\left(\tau_{i j}+\nu \frac{\partial u_{i}}{\partial x_{j}}\right) .
\end{aligned}
$$

The dynamical quantities, $u_{i}, p$ are resolved-scale (filtered) velocity and pressure respectively and $\tau_{i j}$ is the subgrid-scale (SGS) Reynolds stress. $u_{1}, u_{2}$ and $u_{3}$ are respectively the streamwise, lateral and vertical velocity components. $\delta_{i 1}$ is the Kronecker-delta and $\nu$ is the kinematic viscosity. $\partial<P>/ \partial x_{1}$ is the driving force, a constant streamwise pressure gradient. Some numerical experiments were conducted by imposing a fixed pressure gradient (i.e. a body force in each cell) or, alternatively, by specifying a pressure drop between inlet and outlet or by specifying a constant mass flux. Nearly identical results were obtained, with small discrepancies (particularly within the canopy) partly a result of the finite and not always equal averaging times. It was concluded that imposing a constant pressure gradient on every cell as the driving force is indeed a valid method, with fully periodic boundary conditions on the inlet and the outlet. The Smagorinsky SGS model was used with $C_{s}=0.1$, which is recommended by (Shah, 1998) for flow past a blunt obstacle. Note that the Smagorinsky model is widely used by researchers to simulate this kind of flow, with considerable 
success (Shah, 1998; Hanna et al., 2002; Addad et al., 2003; Stoesser et al., 2003). Shah (1998) and Stoesser et al. (2003) also compared the Smagorinsky model with the dynamic model for flow over bluff obstacles. They found that the overall influence of the SGS model is small, at least at low Reynolds number. In the current paper, we report what happens at higher Reynolds numbers when the Smagorinsky model is used for such flows.

In the $x_{1}$ and $x_{2}$ directions the flow is periodic. At the top of the domain, stress free conditions are imposed on $u_{1}$ and $u_{2}$ :

$$
\frac{\partial u_{1}}{\partial x_{3}}=\frac{\partial u_{2}}{\partial x_{3}}=0 ; \quad u_{3}=0 .
$$

The top boundary condition here is simple but widely used (Andrén et al., 1994). It is essentially that for a symmetry plane, so the flow can be considered as one half of a very-rough-wall channel flow. In this respect it is different to a boundary layer flow, but the emphasis here is on the near surface region which, as will be demonstrated, is not too dependent on the outer region of the flow.

The wall model is generally an important issue for LES, and is no less important than the SGS model if the computational cost is to be minimised. For cases where the fine eddies in the vicinity of the wall are of interest, it is recommended that $z_{1}^{+}$is of order of unity $\left(z_{1}^{+}\right.$is the distance in wall unit of the centroid of the first cell from the wall). Note, however, that for a complex geometry like the present one, where separation and attachment processes occur, it is impossible to satisfy this criteria everywhere. We argue here that it is in fact not necessary, at least for obtaining overall surface drag and element-scale dynamics, which turn out to be dominant (see later).

The local wall shear stress is then obtained from the laminar stressstrain relationships:

$$
u^{+}=\frac{u}{\hat{u}_{\tau}}, \quad z^{+}=\frac{\rho \hat{u}_{\tau} z}{\mu}, z^{+}=u^{+},
$$

where $\rho \hat{u}_{\tau}^{2}$ is the local wall shear stress. However, if the near-wall mesh is not fine enough to resolve the viscous sublayer, for simplicity it is assumed that the centroid of the first cell from the wall falls within the logarithmic region of the boundary layer,

$$
\frac{u}{\hat{u}_{\tau}}=\frac{1}{\kappa} \ln E\left(\frac{\rho \hat{u}_{\tau} z}{\mu}\right),
$$

where $\kappa$ is the von Karman constant and $E$ is an empirical constant. The log-law is employed when $z^{+}>11.2$, similar to the criterion used by (Werner \& Wengle, 1991) (11.8) in their model. 
These wall models are relatively simple ones among a number of possible ones representing an impermeable wall (Sagaut, 2001, pp. 238243). Note that the models do not need the averaged tangential velocity and/or wall shear stress, which is an advantage in computing flows with complex geometry. Kanda et al. (2004) also took the local profile of the tangential velocity component to be logarithmic at all solid surfaces, and obtained reasonable results. Comparisons of our LES results with measurements and DNS in section 4 confirm that these simple wall models are effective. Since logarithmic layers are anyway very unlikely to exist on most of the body surfaces this suggests that the flows are not crucially dependent on the near wall regions but, rather, are dominated by the dynamics of the body-scale motions. This marks a crucial difference between these kinds of flows and those over smooth walls at similar Reynolds numbers.

\subsection{Reynolds-AVERAged Navier-Stokes model}

The continuity and momentum equations for steady incompressible are given as follows,

$$
\begin{aligned}
\frac{\partial U_{i}}{\partial x_{i}} & =0 \\
\frac{\partial U_{i} U_{j}}{\partial x_{j}} & =-\frac{1}{\rho}\left(\frac{\partial P}{\partial x_{i}}+\delta_{i 1} \frac{\partial<P>}{\partial x_{1}}\right)+\frac{\partial}{\partial x_{j}}\left(\nu \frac{\partial U_{i}}{\partial x_{j}}-\overline{u_{i}^{\prime} u_{j}^{\prime}}\right),
\end{aligned}
$$

where $U_{i}$ and $P$ are the Reynolds-averaged velocity in the $x_{i}$ direction and the pressure respectively. $\partial\langle P\rangle / \partial x_{1}$ is again the driving force, a constant streamwise pressure gradient. $\nu$ is the molecular kinematic viscosity and $\overline{u_{i}^{\prime} u_{j}^{\prime}}$ are the Reynolds stresses, which must be modelled to close the momentum equations. As is well known, $k-\epsilon$ models (SKE, MKE) apply the Boussinesq type of eddy viscosity approximation,

$$
-\overline{u_{i}^{\prime} u_{j}^{\prime}}=\hat{\nu}\left(\frac{\partial U_{i}}{\partial x_{j}}+\frac{\partial U_{j}}{\partial x_{i}}\right)-\frac{2}{3} k \delta_{i j},
$$

where $\hat{\nu}$ is kinematic viscosity and $k=0.5 \overline{u_{i}^{\prime} u_{i}^{\prime}}$ is the turbulence kinetic energy (TKE), whereas Reynolds stress models (RSM) solve transport equations for the Reynolds stresses.

Because of the standard nature of the RANS models used, more details of the SKE, MKE and RSM models are not given here, other than the values of the constants. For SKE these are as follows,

$$
C_{\mu}=0.09, C_{1 \epsilon}=1.44, C_{2 \epsilon}=1.92, \sigma_{k}=1.0, \sigma_{\epsilon}=1.3 .
$$

However, it is well known that high streamwise strain rates, which must always occur in certain regions of the flow around a bluff body, lead to 
enhanced turbulence energy dissipation in a way that is not modelled by the SKE, which usually therefore returns unphysically high values of turbulence energy in such regions. The standard modification proposed to "fix" this problem within the $k-\epsilon$ context (Leschziner \& Rodi, 1981 ) is to modify the modelling of the production of dissipation term in the $\epsilon$ equation from $C_{1 \epsilon} P \epsilon / k$ (where $\Psi$ represents the generation of turbulence kinetic energy) to

$$
P_{\epsilon}=\left[C_{1 \epsilon}^{\prime} \Psi-C_{1 \epsilon}^{\prime \prime} \hat{\nu} S_{n s}^{2}\right] \frac{\epsilon}{k},
$$

where the constants $C_{1 \epsilon}^{\prime}$ and $C_{1 \epsilon}^{\prime \prime}$ are 2.24 and 0.8 respectively. $S_{n s}$ is the "shear strain" in the direction of streamline. Note that $C_{1 \epsilon}^{\prime}-C_{1 \epsilon}^{\prime \prime}=$ $C_{1 \epsilon}=1.44$, and the scheme reduces to the standard $k-\epsilon$ model for the pure shear-strain case of $\hat{\nu} S_{n s}^{2}=\hat{\nu} S^{2}=\Psi$. However, in threedimensional flows the "shear strain" in the direction of streamline is not well-defined. We followed previous work (Apsley et al., 1995; Castro et al., 1999) in assuming that the most appropriate direction is that of the component of the gradient of local streamwise velocity normal to the local velocity. In the current paper, this modified model is labelled 'MKE'. The other closure constants of MKE are kept the same as those of SKE.

The nine closure constants for RSM (Gibson \& Launder, 1978) are as follows,

$$
\begin{aligned}
& C_{\mu}=0.09, C_{1 \epsilon}=1.44, C_{2 \epsilon}=1.92, C_{1}=1.8, \\
& C_{2}=0.6, C_{1}^{\prime}=0.5, C_{2}^{\prime}=0.3, \sigma_{k}=1.0, \sigma_{\epsilon}=1.3 .
\end{aligned}
$$

Note that the linear pressure-strain model is used for the pressure-strain term, and the generalized gradient-diffusion model (Daly \& Harlow, 1970) is used for the diffusion term in the RSM.

A non-equilibrium wall function (Kim \& Choudhury, 1995) is more suitable for use in complex flows involving separation, attachment and impingement, since the mean flow is subjected to large pressure gradients and thus changes rapidly. The key improvements in the nonequilibrium wall function used here are: (1) Launder and Spalding's log-law for mean velocity is sensitized to the pressure-gradient effects; (2) the two-layer-based concept is adopted to compute the budget of turbulence kinetic energy in the wall-neighboring cells. The nonequilibrium wall function was applied for all the three RANS models, SKE, MKE and RSM.

Many of the initial LES computations were undertaken using three quite different, commercially available CFD codes. Considerable time was spent in assessing the performance of these codes and comparing results of computations from all three codes using identical grids. We 
emphasise that the results turned out to be essentially independent of the code used, provided that each was implemented correctly and, crucially, that the numerical discretisation scheme in both time and space was second order. However, we found that because of the different solution strategies used, the codes varied significantly in the efficiency with which the LES computations were performed; we are not concerned here with that issue. Whilst most of the results presented here were obtained using one particular code, this should not be taken as an overall endorsement of that code over the others nor as an indication that it was particularly efficient. The same code was used for the RANS computations but, again, essentially identical results could be obtained using the alternative codes.

\section{Further Numerical Details and Settings}

The CFD code is a finite-volume (FVM), unstructured-grid code. For the baseline case C20S (Figure 1a) only uniform mesh $(\Delta x=\Delta z=\Delta z)$ or hexahedral cells with two or three levels of grid refinements with hanging nodes were used. In the latter case, the mesh in the vicinity of the cubes and the bottom wall was uniform.

In addition to this baseline case (C20S) a similar array of random height bodies (RM10S) was studied (see Figures 1b,c). The black dots in Figure 1 denote sampling locations at which experimental, DNS and LES data are compared. The computational domain for C20S was $L_{x} \times$ $L_{y} \times L_{z}=4 h \times 4 h \times 4 h$, where $h=0.02 m$ is the height of cube. Coceal et al. (2006) mostly used this domain size in their DNS of the same flow, but for validation they also employed domain sizes of $8 h \times 8 h \times 4 h$, $4 h \times 4 h \times 6 h$ and $16 h \times 12 h \times 8 h$. Although a $4 h \times 4 h \times 4 h$ domain with periodic boundary conditions was, not surprisingly, found to be too small to capture all of the largest scales of turbulence - particularly the very long 'streaky' structures near the surface - they found that the differences in mean velocity and turbulence statistics were negligible except, not surprisingly, in the vicinity of the top boundary. For the present RM10S case a domain of $L_{x} \times L_{y} \times L_{z}=16 h_{m} \times 16 h_{m} \times 10 h_{m}$ was used, where $h_{m}=0.01 \mathrm{~m}$ was the mean height of the obstacles, so this domain contained 64 obstacles.

For $\mathrm{C} 20 \mathrm{~S}$ at $\mathrm{Re}=5 \times 10^{3}$, a uniform mesh of 0.25 million cells with $16 \times 16 \times 16$ grid points per cube (LES16, medium mesh) was mainly used. In some simulations, a more refined mesh of 0.75 million cells with $32 \times 32 \times 32$ grid points per cube (LES32, fine mesh, see Figure 2a) was used, whereas some other simulations were done with a uniform mesh of only 31 thousand cells with $8 \times 8 \times 8$ grid points per cube (LES 8 , coarse 


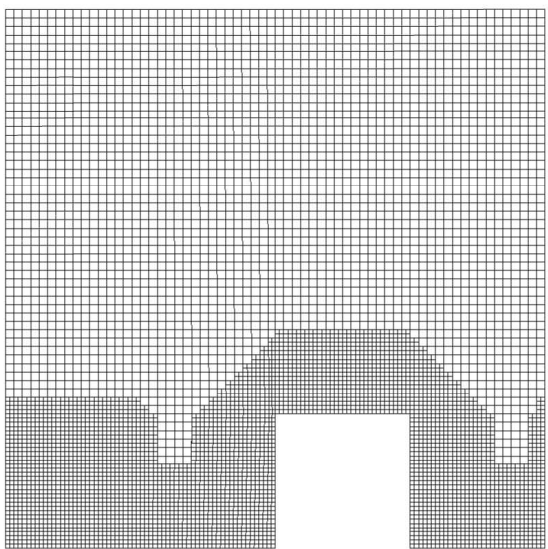

(a)

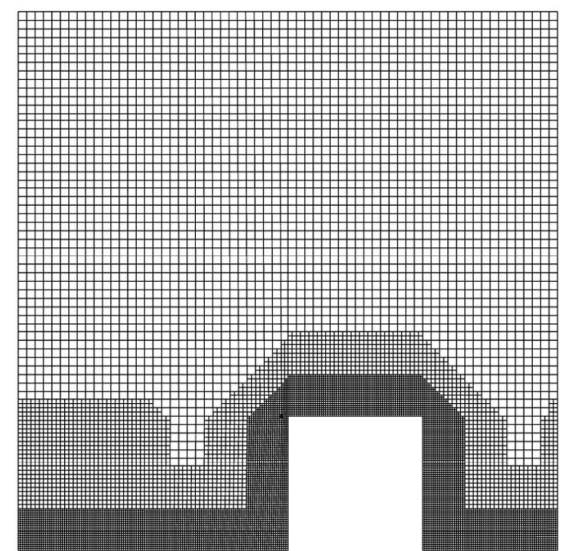

(b)

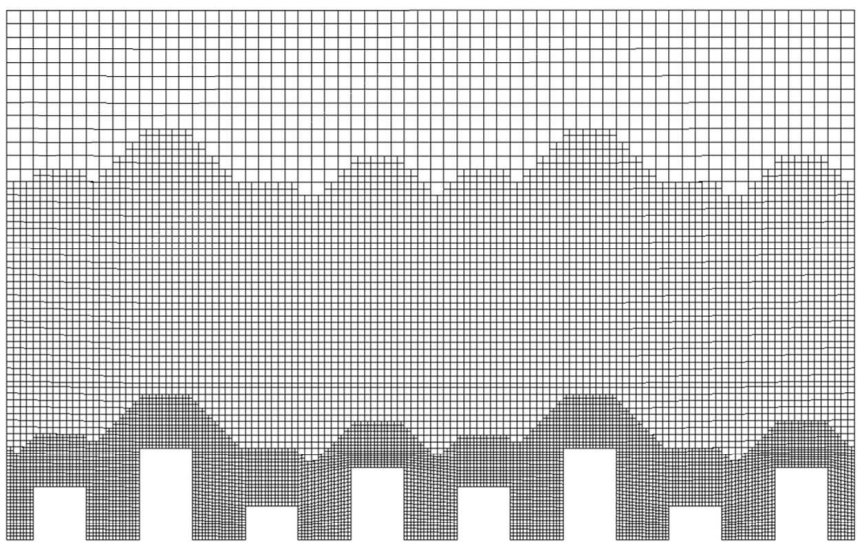

Figure 2. (a): mesh with finest resolution $h / 32$ for C20S (LES32) at $\mathrm{y}=0.5 \mathrm{~h}$; (b): mesh with finest resolution $h / 64$ for C20S (LES64) at $\mathrm{y}=0.5 \mathrm{~h}$; (c): mesh with finest resolution $h / 16$ for RM10S at $\mathrm{x}=5 h_{m}$.

mesh). The maximum CFL number in all the simulations was less than unity. The distances in wall units of the centroid of the first cell from the wall, $z_{1}^{+}$, for LES32 (fine mesh) and LES16 (medium mesh) were approximately 6.3 and 12.6 respectively, based on the global friction velocity $u_{*}=0.89 u_{\tau}$ (see equation 6 ). However, the distances in wall units of the centroid of the first cell from the wall $z_{l 1}^{+}$based on the local friction velocity were much lower, because obstacle form drag provides the dominant part of the total drag, as discussed in later. The final calculated $z_{l 1}^{+}$for LES16 varied from 8 at the leading edge on the top roof of the cube to less then 2 behind the cube on the bed of the channel, with an average value of approximately 5.6. 
For $\mathrm{C} 20 \mathrm{~S}$ at $\mathrm{Re}=5 \times 10^{4}$, a three-level mesh of about three million cells with $64 \times 64 \times 64$ grid points per cube in the near wall region was used (LES64, see Figure $2 b$ ). At Re $=5 \times 10^{6}$, only the mesh LES16 was used. For RM10S, a three-level mesh of 2.3 million cells with $16 \times 16 \times 16$ grid points per $h_{m} \times h_{m} \times h_{m}$ in the near wall region was used (see Figure 2c).

The fluid density and the kinematic viscosity for $\mathrm{C} 20 \mathrm{~S}$ at $\mathrm{Re}=5000$ were set to $1.205 \mathrm{~kg} \mathrm{~m}^{-3}$ and $1.5 \times 10^{-5} \mathrm{~m}^{2} \mathrm{~s}^{-1}$, respectively, and the constant pressure gradient in equation 1 was set to $-1.59 \mathrm{~Pa} \mathrm{~m}^{-1}$. The bed stress can be obtained from:

$$
\rho u_{\tau}^{2}=\frac{\partial P}{\partial x_{1}} \times L_{z},
$$

where $u_{\tau}$ is by definition the total wall friction velocity. The roughness Reynolds number, $R_{\tau}=u_{\tau} h / \nu$, was 433 , which is in the fully rough regime. Higher $R e$ was achieved simply by changing the value of the kinematic viscosity appropriately, with other variables unchanged. For RM10S, the kinematic viscosity was set to $1.67 \times 10^{-5} \mathrm{~m}^{2} \mathrm{~s}^{-1}$, the constant pressure gradient was set to $-5.14 \mathrm{~Pa} \mathrm{~m}^{-1}$.

Although individual velocity profiles above the canopy do not generally contain a logarithmic region, it has been shown (Cheng \& Castro, 2002; Coceal et al., 2006) that spatially averaging the velocity at each height does yield the usual rough-wall log-law expressed by,

$$
U(z)=\frac{u_{*}}{\kappa} \ln \left(\frac{z-d}{z_{0}}\right),
$$

where $\kappa$ is the von Karman constant, $u_{*}$ is the friction velocity, $d$ is the zero-plane displacement and $z_{0}$ is the roughness length. It is not obvious how best to fit the spatially averaged streamwise mean velocity to determine the characteristic velocity $u_{*}$ when, as here (and unlike the regular boundary layer) the flow is maintained by a constant pressure gradient. Coceal et al. (2006) noted that the effective $u_{*}$ in a pressuredriven variant of a rough-wall boundary layer is not the same as $u_{\tau}$ and found that a theoretically derived value of $u_{*}=0.89 u_{\tau}$ (for this geometry) gave the best fit for their DNS results, compared with Cheng \& Castro (2002)'s (boundary layer) measurements. Here we also set $u_{*}$ to be $0.89 u_{\tau}$ for all the LES cases.

A second-order backward implicit scheme in time and a secondorder central difference scheme in space were applied. Shah (1998) stresses that non-centred schemes such as the 'upwind' method have built-in dissipation which can lead to damping of the turbulent kinetic energy in LES. Central difference schemes can yield unphysical oscillations; however, this should be taken as an indication of insufficient 
grid resolution and should not be suppressed by the use of numerical dissipation. Note that at the differential level the conservation equation is exactly satisfied on each control volume in the finite-volume method (FVM). For example, the convection terms in the momentum equations are written in the total derivative form, $\frac{\partial u_{i} u_{j}}{\partial x_{j}}$, and the conservation of kinetic energy can then be automatically maintained (Williams, 1969). Little extra treatment is needed for the convection term in the momentum equation. At the discrete level conservation depends on the quality of the mesh, the detailed discretization scheme and the storage arrangements. On the other hand, for finite difference methods (FDM) in LES, various treatments have been used for the convection term (Mason, 1989; Moeng, 1984; Nieuwstadt \& Brost, 1986; Schmidt \& Schumann, 1989). Shah (1998) and Stoesser et al. (2003) also applied the central difference scheme for the convection term in their own FVM LES codes for flows over bluff obstacles. (Note that if a passive scalar equation is solved, extra attention does need to be paid to the convection term, see Xie et al., 2004b, for example).

The initial duration of most of the runs was $200 T\left(T=h / u_{*}\right)$, which was also used in the DNS (Coceal et al., 2006), whereas the subsequent averaging duration for all the statistics was normally $80 \mathrm{~T}$, less than that used in the DNS (400T). We noticed that the variation in statistical data throughout the roughness sub-layer (including the canopy region) was usually small once the averaging duration exceeded about $20 T$, provided the initialising duration was sufficiently long. (200T was generally adequate). However, the variations in the streamwise mean velocity at the top of domain was not small; this will be addressed in section 4.1 .

A second-order upwind difference scheme in space was applied for the SKE, MKE and RSM (RANS) models. In the vicinity of all the solid walls four layers of a fine mesh having $32 \times 32 \times 32$ grid points per cube were used, whereas in the other regions a medium mesh with $16 \times 16 \times 16$ grid points per cube was used. Both grids had some 0.36 million cells. Other numerical settings are the same as those for the large-eddy simulations.

We conclude this section by commenting on the required computer time for typical computations. As an example, the numerical simulation of the regular cube array using about $750 \mathrm{~K}$ cells, when performed on a local Linux PC cluster system using only 16 (2.2GHz 64-bit AMD) processors, provided converged statistics within about 100 hours, when started from an initial coarser mesh solution which itself took about 21 hours. This is a factor of roughly 40 longer than required to obtain a converged RANS solution on the same mesh. The more expensive 
LES computations for the RM10S (random) array on a grid of about 2.5 million cells required about 20 days ( 7680 hours) on 16 processors. Increasing the number of processors reduces the time required (roughly linearly).

\section{Results and Discussions}

\subsection{Some LES RESUlts COMPared With DNS AND LDA}

Vertical profiles of streamwise mean velocity at four typical horizontal positions obtained using the coarse, medium and fine meshes are shown in figure 3 and are in satisfactory agreement with both the DNS data (DNS64 CTBC - Coceal et al., 2006) and wind tunnel measurements (LDA CC - Castro et al., 2005). Note that the resolution of the DNS is $h / 64$ (recall $h$ is the cube height). However, LES8 underestimates the streamwise mean velocity for $z / h<1$ by over $25 \%$ in the gap, Figure 3d, which is simply due to the lack of resolution. Visualization shows that LES8 fails to predict a reverse flow (separation bubble) on the two lateral sides of each cube, with the consequence that the streamwise velocity at the middle of the gap is underestimated. Stoesser et al. (2003) also noticed an underestimation of the streamwise mean velocity in the gap. They did not analyze it further in their paper, but suggested that it was because that the recovery of the flow behind the obstacle is underestimated.

Figure 4 shows comparisons of turbulence intensities behind the cube using the coarse, medium and fine meshes and data from DNS and measurements. Again, the differences between the results obtained using the three meshes are relatively small. However, figure $4 \mathrm{c}$ shows that both DNS64 and LES32 have successfully captured the peak $w_{r m s}$ around the height of the cube, which suggests that a fine mesh (or at least a stretched grid having greater resolution in that region) is needed to simulate accurately the details of the shear layer above the canopy. All the simulations underestimate the lateral fluctuation, $v_{r m s}$, compared with measurements (figure $4 \mathrm{~b}$ ). Since DNS with a significantly larger domain size yielded a similar underestimation, neither the small domain size nor the simple wall model used for the present LES can be blamed for this apparent deficiency. Note that in the vicinity of the bottom wall there is a sharp peak of $v_{r m s} / u_{*}(4 \mathrm{~b})$ for LES8 and LES16; this is almost certainly caused by too coarse a resolution.

A strong shear layer can be seen in Figure 4a at the height of cube, which is consistent with the data in Figure $3 \mathrm{~b}$. All the simulations successfully captured this shear layer, although note again that the 

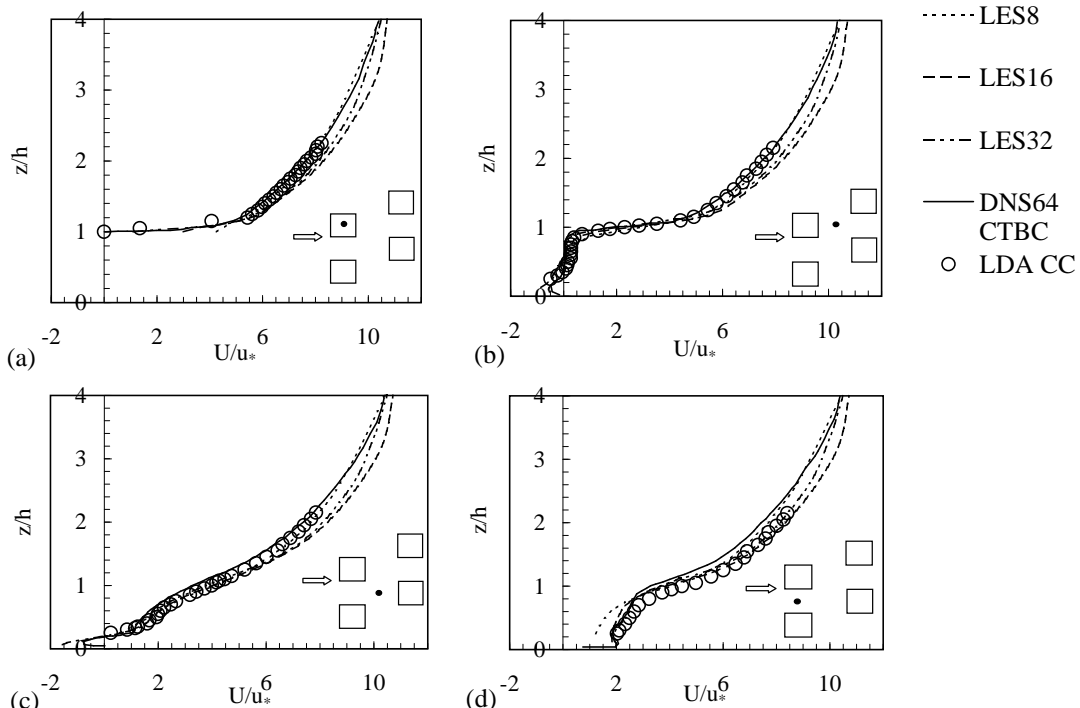

(c)

$\mathrm{U} / \mathrm{u}^{6}$

(d) ${ }^{-2}$

Figure 3. Vertical profiles of streamwise mean velocity at four horizontal positions. (a): over cube; (b): behind cube; (c): in front of cube; (d): in gap.

coarse and medium meshes failed to capture the peak in $w_{r m s}$. As discussed later the features of flows above and within an array of cubes depend on the cube layout - staggered or aligned arrangements leading to significant differences. Also, for a large enough depth $(\delta / h)$ the flow tends to be more like a rural boundary layer, generating an evident plateau of shear stress and turbulence intensities in the near-surface layer. On the other hand, with a smaller $\delta / h$ (as here), the flow becomes more like an urban boundary layer where the size of the large eddies generated by the obstacles is a much larger fraction of the flow depth. Figure 5 shows comparisons between turbulence intensities in front of a cube. The differences among the coarse, medium and fine mesh results are again generally small, especially above the canopy. Note that only LES8 shows a rise in $v_{r m s}$ near the wall (figure $5 \mathrm{~b}$ ) which is, again, very likely due to the low resolution. Also in figure $5 \mathrm{c}$, there is a weak resolution dependence within the canopy.

Figure 6 shows comparisons between the turbulence intensities in the gap between the cubes. All the LES data are in reasonable agreement with the measurements and the DNS data. Nevertheless, the coarse grid results (LES8) fail to capture the peak in the vertical fluctuations at the height of the cube in figure 6c. 

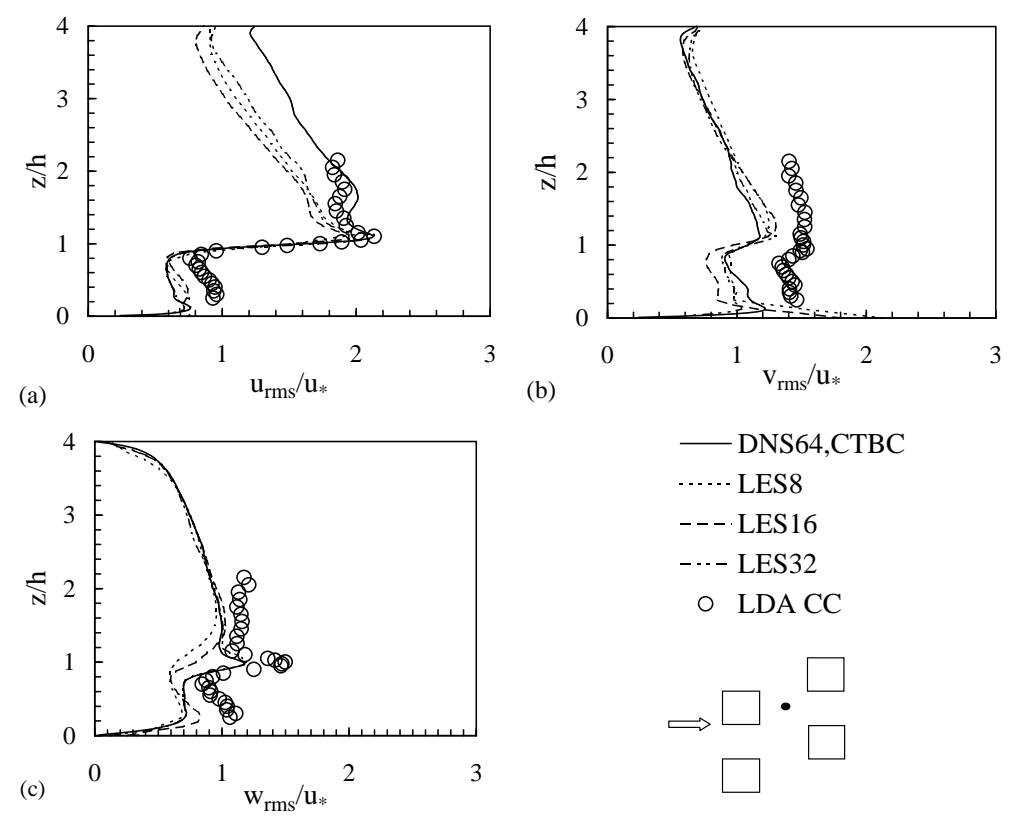

Figure 4. Vertical profiles of turbulence intensities behind cube.

Figure 7 shows comparisons of vertical profiles of Reynolds shear stress from the LES16 results, Castro et al. (2005)'s wind tunnel data and Coceal et al. (2006)'s DNS results. The comparison is very encouraging. A linear variation of Reynolds shear stress with height is evident for LES and DNS data, which is due to the constant driving body force imposed in the numerical simulations. In figure 7c LDA measurements, DNS64 and LES16 data all yield a zero shear stress around $z=0.3 h$. Note that, as seen in figure 3 and in figure 8 (at the centre of the circulation in front of cube), the mean streamwise velocity but not the gradient of mean streamwise velocity is also approximately zero at $z=0.3 h$. DNS64 captures a peak at approximately $0.1 \mathrm{~h}$ (figure $7 \mathrm{c}$ ), presumably because the laminar sublayer is adequately resolved.

Figure 8 shows mean velocity vectors of $(u, w)$ in the $x-z$ symmetry plane through a cube obtained using LES and DNS (Coceal et al., 2006), in which the DNS vectors are plotted at reduced resolution for greater clarity. The two plots are qualitatively identical in that both clearly reveal the location of the stagnation point, the location and the size of the clockwise circulation at the corner in front of the cube, 

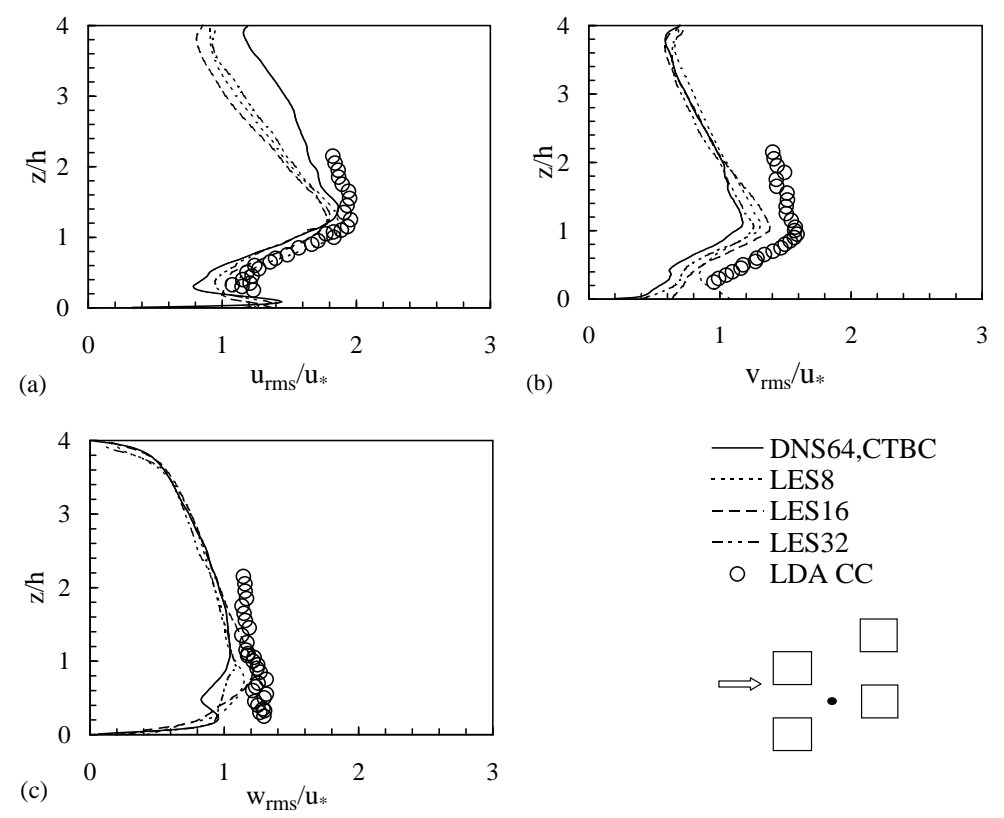

Figure 5. Vertical profiles of turbulence intensities in front of cube.

the reverse updraft flow behind the cube, and flow pattern on top roof of the cube behind the leading edge. In regard to the latter, there is little evidence of any reversed flow region just aft of the leading edge. However, reducing the turbulence levels just upstream of the element increases the size of the separation bubble on the top roof of the cube behind the leading edge. So Cheng et al. (2003) and Meinders \& Hanjalić (1999), for example, examined a case of aligned cubes with a much lower area density of $6.25 \%$, yielding lower turbulence levels at element height, and found a thin reverse mean flow on the top roof of the cube behind the leading edge. In contrast, neither the DNS nor the present LES32 generated a reverse mean flow in this region, because the staggered cube case with a larger area density $25 \%$ generates stronger turbulence mixing in this region. Also note that there are two primary separation points on $z=0$. These are at $\Delta x \approx 0.73 h$ ahead of the cube and $\Delta x \approx 0.93 h$ behind the cube. A secondary separation occurs at $\Delta x \approx 0.06 h$ ahead of the cube, which is comparable with that found at $\Delta x \approx 0.05 h$ in flow over a single cube (Shah, 1998). The medium mesh LES16 generated a nearly identical mean velocity vector field 

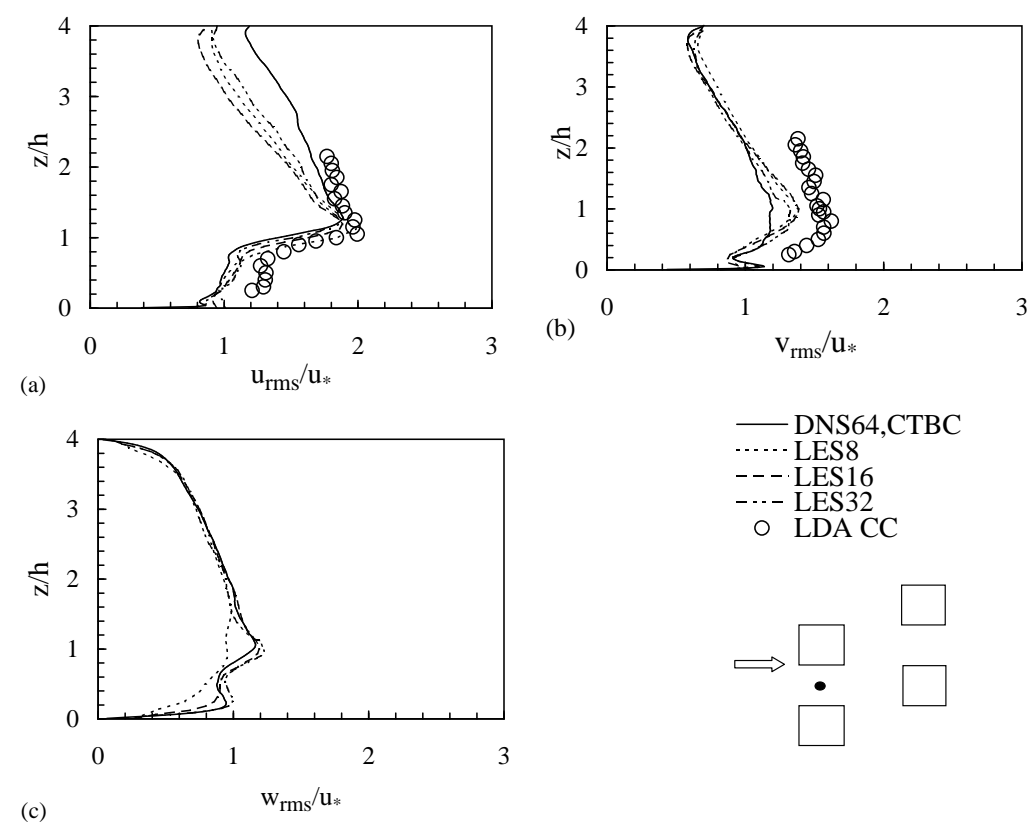

(c)

Figure 6. Vertical profiles of turbulence intensities in gap.

as the fine mesh LES32 although it failed to predict the secondary surface separation near the upstream corner. Consequently a greater downward flow in the near wall region ahead of the cube was generated, which postponed the primary separation point on $z=0$ to occur at $\Delta x \approx 0.9 h$ ahead of the cube. However, this subtle discrepancy made little difference to the mean pressure distribution on the cube and thus the total surface drag, see later, because the velocities near the bottom surface are so low.

Similarity parameters of turbulent flow over rough surfaces in neutral conditions are summarized in Table I, which again confirms that the LES generally performs well against the finer resolution DNS. Note that the lowest values are seen for cases in which the velocity profiles have an inflection point, whilst the other data are typical values in the near-surface layer of more classical rough-wall boundary layers (which have much larger $\delta / h$ ). The table suggests that the flow over and around staggered cubes quite likely has dynamics determined by the mixing-layer-type velocity profiles around the cube height - i.e. a profile with an inflection point - as argued by Finnigan (2000) in 

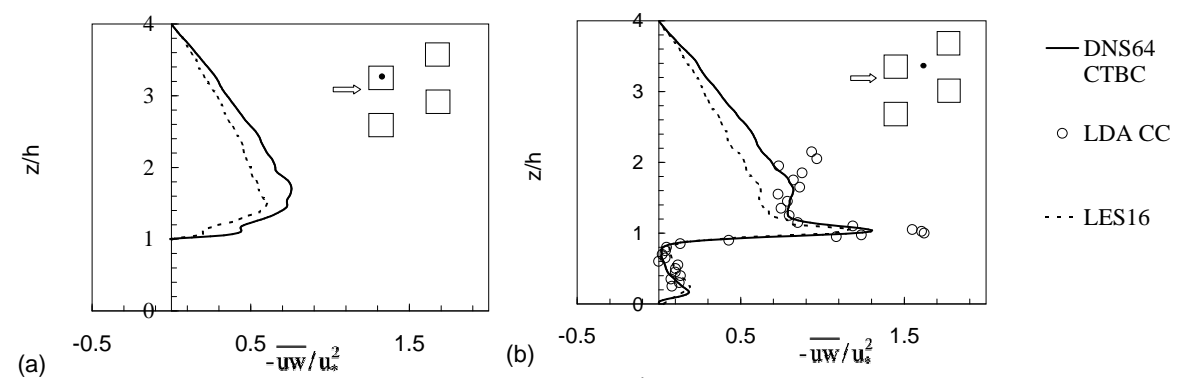

(a)

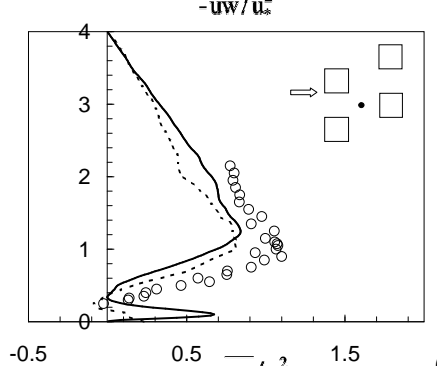

(b)

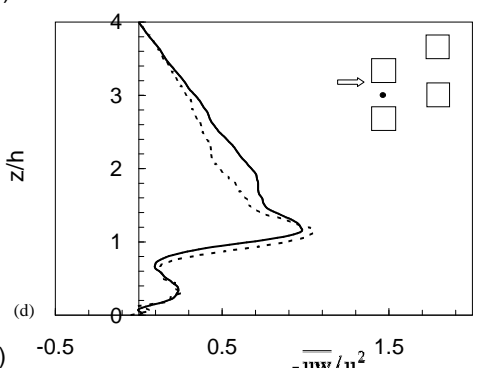

Figure 7. Vertical profiles of Reynolds shear stress at four horizontal positions. (a)over cube, (b)behind cube, (c)in front of cube, (d) in gap.

Table I. Comparison of similarity parameters of flow over rough surface

\begin{tabular}{lllll}
\hline $\begin{array}{l}\text { Maxima for staggered-cubes, } \\
\text { mixing layer \& canopy flows }\end{array}$ & $u_{r m s} / u_{*}$ & $v_{r m s} / u_{*}$ & $w_{r m s} / u_{*}$ & $\begin{array}{l}\text { Inflection } \\
\text { in U(z) }\end{array}$ \\
\hline LES16 & 1.84 & 1.31 & 1.05 & Yes \\
DNS(Coceal et al., 2006) & 1.93 & 1.23 & 1.05 & Yes \\
Mixing layer(Finnigan, 2000) & 1.8 & $/$ & 1.4 & Yes \\
Plant canopy(Finnigan, 2000) & $1.8-2.0$ & $/$ & $1.0-1.2$ & Yes \\
\hline Values in the 'classical' surface & layer & & & \\
LES (Xie et al., 2004a) & 2.7 & 1.38 & 1.14 & No \\
Experiment (Xie et al., 2004a) & 2.4 & 1.6 & 1.2 & No \\
Fackrell \& Robins (1982) & 2.3 & 1.6 & 1.2 & No \\
Stull (1993) & $2.47,2.49$, & $1.70,1.73$, & $1.0,1.3$, & \\
& 2.55 & $2.07,2.47$ & 1.58 & No \\
\hline
\end{tabular}




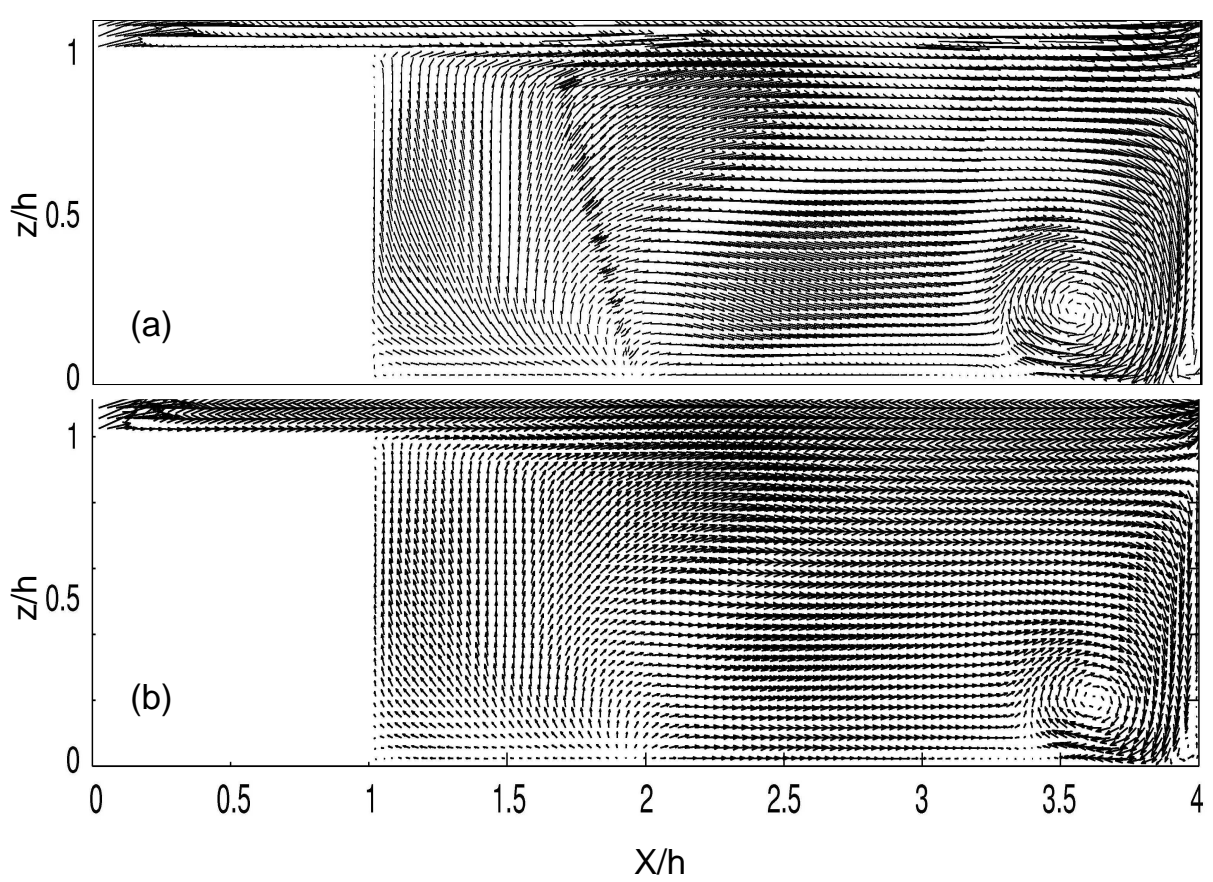

Figure 8. Mean velocity vectors in the $\mathrm{x}-\mathrm{z}$ plane at $y / Z_{H}=1.5$. (a): LES32, fine mesh; (b): DNS64 (every two vectors plotted). Note that for the two cases different plotting routines were used, having small differences in arrow geometry.

the plant canopy flow case. In the case of an aligned array of cubes, which has 'smooth' channels within the canopy for flow to pass through, Kanda et al. (2004) and Cheng \& Castro (2002) found that the drag forces are less than those on staggered cubes and the flow within the canyon is apparently more similar to that near the surface of a regular channel or boundary layer. Thus, not surprisingly, the largest values in the horizontally averaged stresses $\left(u_{r m s} / u_{*}, w_{r m s} / u_{*}\right)$ were observed to be close to the bed ((Kanda et al., 2004)) whereas, in contrast, the peak stresses occur near the roof level for the present case of a staggered cube situation. 

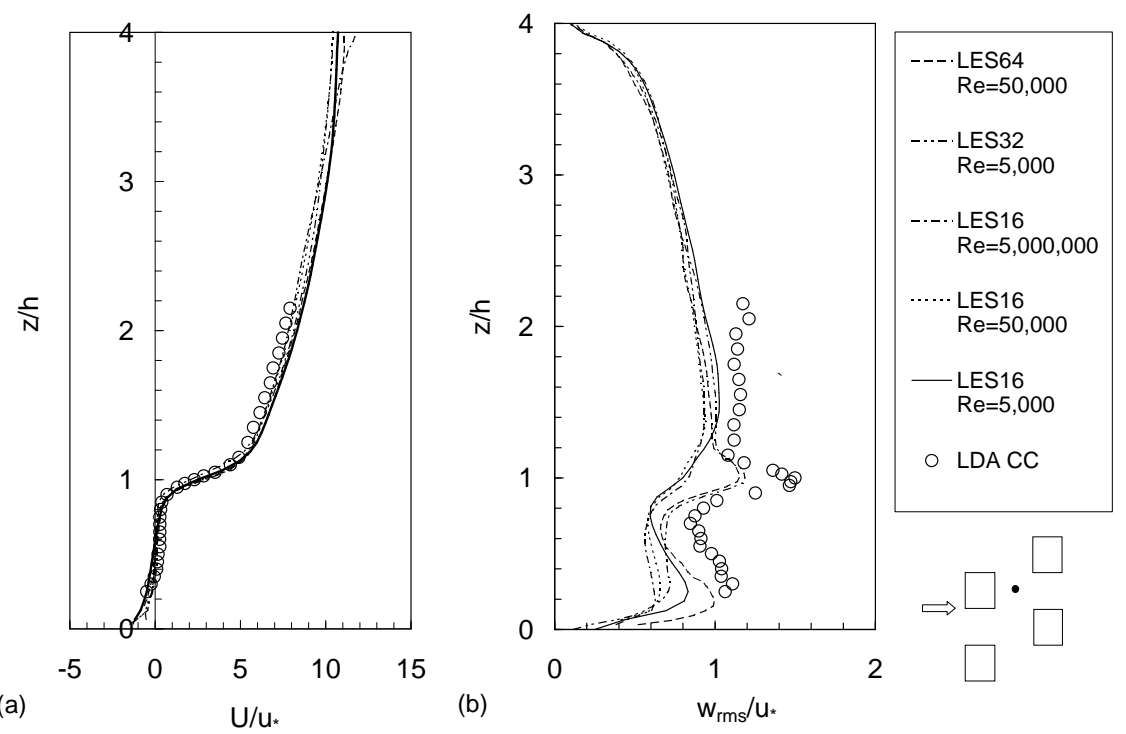

Figure 9. Vertical profiles of turbulence statistics at various Reynolds numbers. (a): streamwise mean velocity; (b): turbulence intensity.

\subsection{LES FOR FLOWS AT HIGHER REYNOLDS NUMBERS AND FLOWS OVER RANDOM GEOMETRY}

Test case C20S at $R e=5 \times 10^{4}$ with meshes LES16 and LES64 (see figure $2 \mathrm{~b}$ ) and at $R e=5 \times 10^{6}$ with mesh LES16 were simulated. Figure 9 shows one typical plot - the vertical profile of streamwise mean velocity and the rms of vertical velocity. The LES64 at $R e=50,000$ (like LES32 for $R e=5000$, Figure 4c yields a peak in the shear layer at the cube height (figure 9b) whereas LES16 does not resolve this at any $R e$. The discrepancy in $w_{r m s}$ at $z=0.2 h$ is due to inadequate mesh resolution, as was clearly seen for $R e=5000$ in Figure 4 . The other turbulence statistics data at various Reynolds number show similar results. It is emphasised again that the flow around the obstacle below some height, e.g. $z=0.3 h$, is very weak and hence contributes little to the total drag force of the obstacle. It is significant that for $R e=50,000$ what might be considered a relatively coarse mesh, LES64, captures the shear layer peak, just as does LES32 for $R e=5000$. Taken with the experimental data (obtained by Cheng \& Castro, 2002 for $5000<R e<$ 13000) the results suggest that any Reynolds number dependency on the turbulence levels in this shear layer is very weak. 


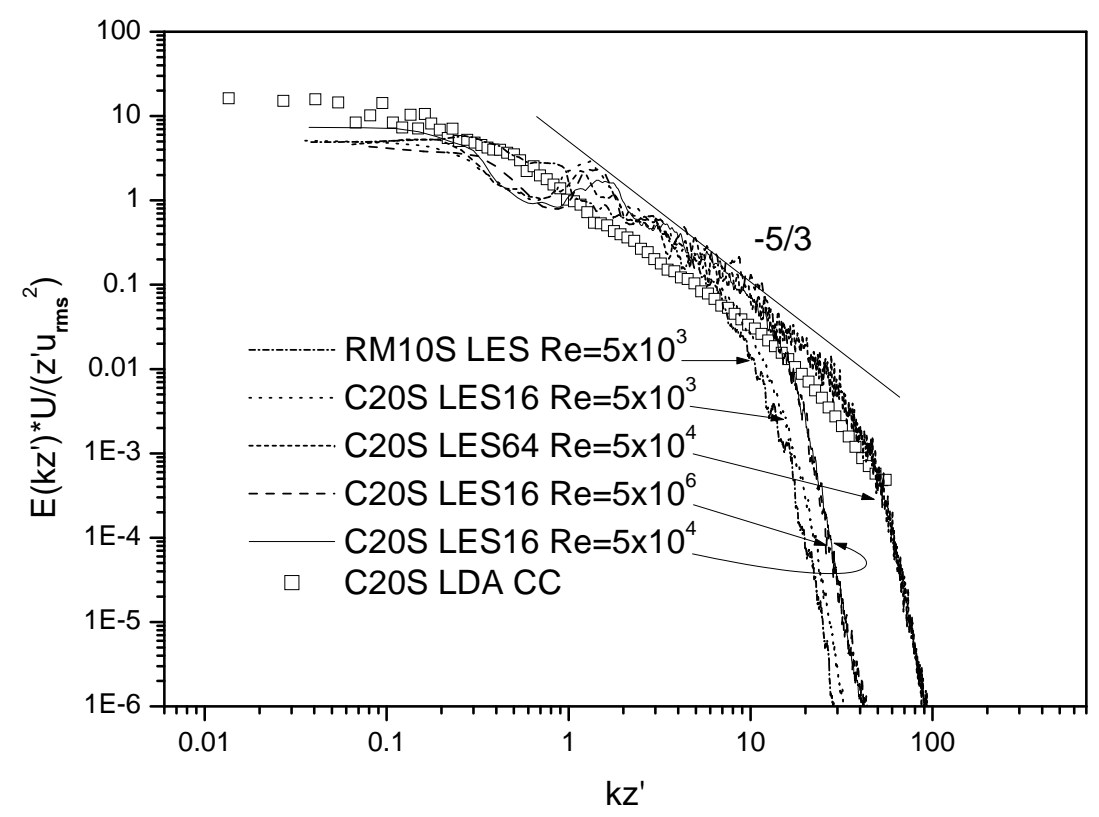

Figure 10. Spectra of the axial turbulence component, plotted in inner-layer scaling.

In discussing the effects of Re and the sub-grid modelling it is helpful to consider spectral data. Figure 10 shows typical spectra, plotted in inner-layer scaling. Note that $k=2 \pi f / U$ is the wavenumber and $z^{\prime}=z-d$, where $d$ is the zero-plane displacement and is $0.013 \mathrm{~m}$ for C20S and 0.012m for RM10S (Cheng \& Castro, 2002 and Castro et al., 2005). The data were sampled at $z=1.5 h$ for C20S, except for the case LES64, $R e=5 \times 10^{6}$ case, for which data were sampled at $z=1.25 h$. For RM10S the data were sampled at $z=3 h_{m}$. A wide (e.g. more than one decade) inertial sub-range with slope $-5 / 3$ was found on the spectrum obtained from measurements (Meinders \& Hanjalić, 1999; Castro et al., 2005) at low Reynolds numbers of approximately 5000 and 13000 , respectively. This again suggests that turbulence generated by urban-like obstacles (with sharp edges) is large-scale dominated.

Figure 10 indicates that all of the numerical simulations resolve an inertial sub-range, but to an extent depending on the resolution. There is a relatively wide inertial sub-range even at the lowest Reynolds number (5000); increasing Re simply extends this range to higher frequencies. This is seen even with the relatively coarse grid LES16 (compare the $R e=5 \times 10^{3}$ and $5 \times 10^{4}$ data in Figure 10). The difference is not 
large enough, of course, and a higher resolution LES64 is required to yield a significantly greater extension to higher frequencies. However, the experimental data indicate that $92 \%$ of the total energy is contained below $k z^{\prime} \approx 5$ and even with LES16 this part of the spectrum is adequately captured. With a further increase in $R e$ to $5 \times 10^{6}$, the simulated inertial sub-range remains quite restricted but given the relative unimportance of these smallest scales in this kind of flow, this seems not to be a serious drawback. The ratios of the subgrid-scale eddy and the molecular viscosity for these various computations range from about 0.1 to a few hundred.

Turning to the low wave-number part of the spectrum, for all the C20S cases there exists an evident distortion in the spectrum near $k z^{\prime}=1$. This is the wave length corresponding to the domain size in the streamwise direction. There is no such distortion in the spectrum of RM10S, for which a much larger domain size was used. Similar effects have been observed by Coceal et al. (2006) and it seems clear that the distortion is caused by the relatively small domain size. Nevertheless, the integrated energy for $k z^{\prime}<2$ is approximately $80 \%$ for RM10S and $70 \%$ for the C20S case at $R e=5000$ with LES16. Given also the $92 \%$ energy content for $k z^{\prime}<5$ in the measurements, mentioned above, and the adequate resolution of at least the upper part of the inertial subrange, this suggests that most of the energy of the large eddies was successfully captured. We again emphasise that in this flow the large eddies (on the order of the obstacle size) are dominant.

Figure 11 plots the vertical profile of the laterally-integrated pressure difference between the front and back faces of a cube, normalized by the pressure difference, $\Delta p_{m}$, between the centre of the front and rear faces. The comparisons for $R e=5 \times 10^{3}$ between the LES16, LES32 and both the DNS data and the measurements is very satisfactory. However, the very coarse mesh (LES8) smoothes much of the peak near the top of the cube. The integrated pressure difference between front and rear faces using the fine mesh (LES32) is about 93\% of the total imposed body force, showing that the form drag is totally dominant, with only a small contribution from the viscous drag. It is also noted that data at higher Reynolds numbers with more than $16 \times 16 \times 16$ grids per cube are in good agreement with DNS data and measurements (which were $R e=13000$ ), again suggesting that any Reynolds number dependency is very weak. For the $R e=5 \times 10^{4}$ and $R e=5 \times 10^{6}$ cases the integrated pressure differences between front and rear faces are all over $87 \%$ of the total body force imposed. Overall, the results suggest that a medium mesh $(16 \times 16 \times 16$ cells per cube) would be sufficient for the simulation of a real urban area, at least as far as obtaining the total drag force is concerned. 


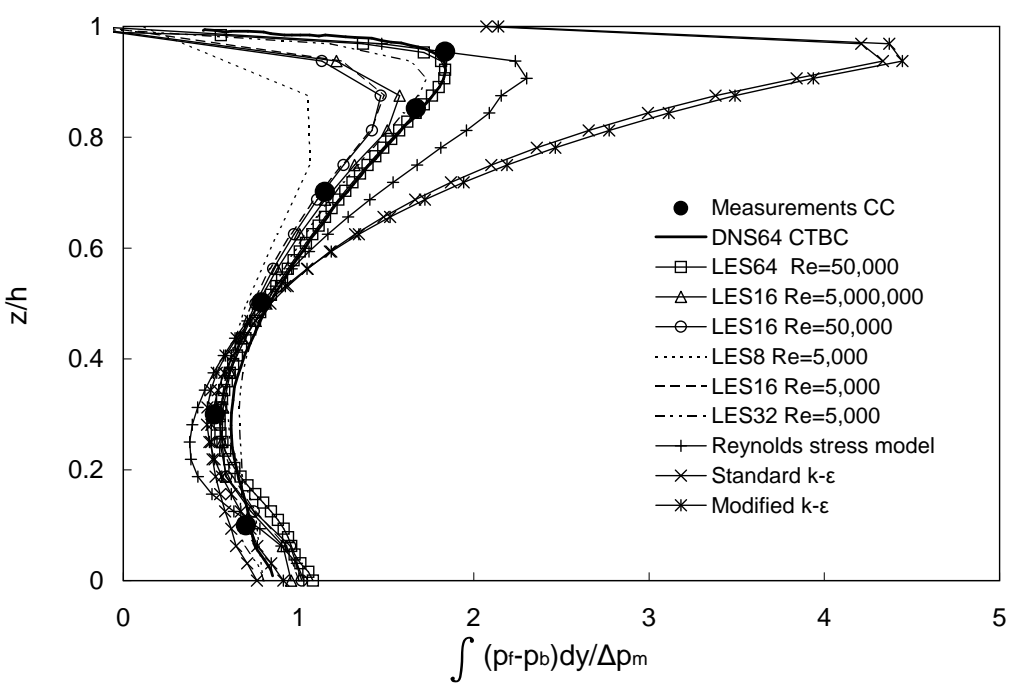

Figure 11. Vertical profiles of laterally-integrated pressure difference between front and back faces of the cube.

Coceal et al. (2006) conducted an intensive domain size test for C20S using a larger domains and found, as noted earlier, that the turbulence statistics up to second order were not significantly affected by domain size. Furthermore, we also conducted an LES for RM10S using a domain size $L_{x} \times L_{y} \times L_{z}=16 h_{m} \times 16 h_{m} \times 10 h_{m}$. Figure 12 shows vertical profiles of streamwise velocity and turbulence intensities behind a $17.2 \mathrm{~mm}$ obstacle in the RM10S array. Note that the measured depths of the boundary layers in the wind tunnel were $141 \mathrm{~mm}$ and $137 \mathrm{~mm}$ for C20S and for RM10S respectively (i.e. $\delta / h=7.0 \& 13.7$ ). Comparing figures 3 and 4 with figure 12, it is emphasised again that both the small domain (C20S) and large domain (RM10S) LES16 computations generated reasonable agreement with experiment at least up to the inertial sublayer (whose upper limits were respectively $46 \mathrm{~mm}$ and 33 $\mathrm{mm}$ in the two cases). This provides further confidence that the current test case C20S is indeed sufficient for an economical benchmark for the LES codes. 

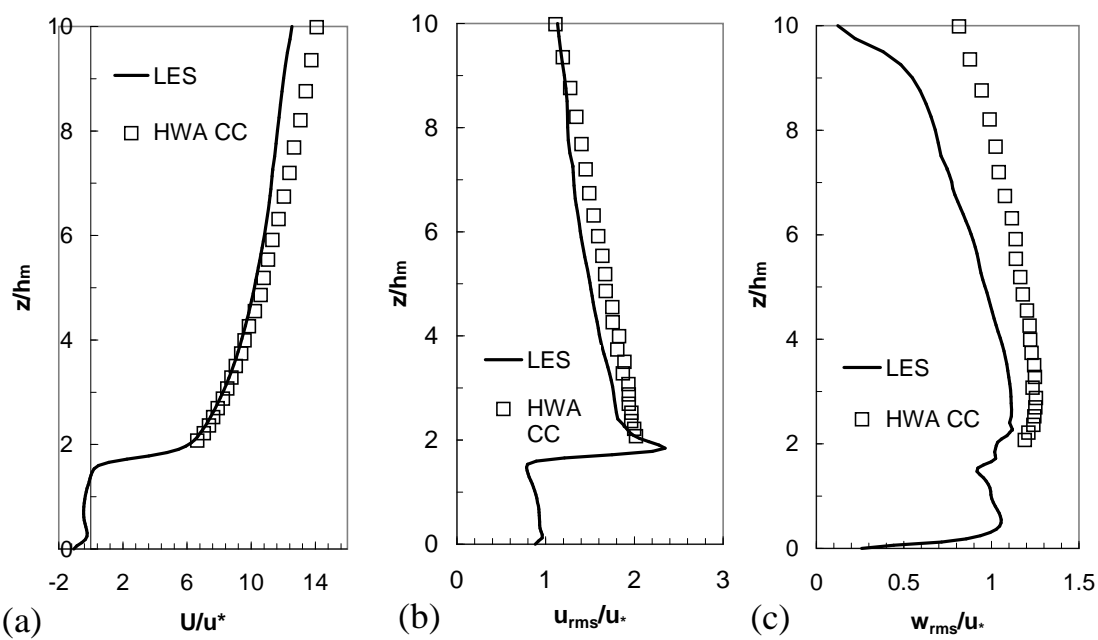

Figure 12. Vertical profiles of streamwise velocity and turbulence intensities behind $17.2 \mathrm{~mm}$ obstacle, RM10S.

\subsection{Reynolds-AVERAged NAVIER-Stokes RESUlts}

Figure 13 shows streamwise velocity and turbulence kinetic energy $\left(T K E=0.5\left(u^{\prime 2}+v^{\prime 2}+w^{\prime 2}\right)\right)$ in the gap between cubes, given by the three RANS models (SKE, MKE and RSM) and the LES using the medium mesh (LES16). At this location (in the gap) the difference between the RANS and LES results was largest (compared with corresponding differences at the other three stations examined - over the centre, behind and in front of the cube). In figure 13a, all the RANS models underestimate the streamwise mean velocity within the canopy and both the SKE and MKE models fail to predict a reverse flow on the lateral sides of the cube. Also, the RSM model underestimates the size of the reverse flow - compared with the LES data (see Figure 14). Underestimation of the reverse flow must be one of the reasons that the streamwise mean velocity within the canopy is underestimated in the lateral gap between the cubes, as noted earlier.

Figure 13b shows that all the RANS models underestimate the $T K E$ in the canopy, when compared with the LES results. It is not surprising that the MKE model predicts a lower TKE than SKE, because MKE is designed to enhance turbulence energy dissipation in the region with high streamwise strain rates. 


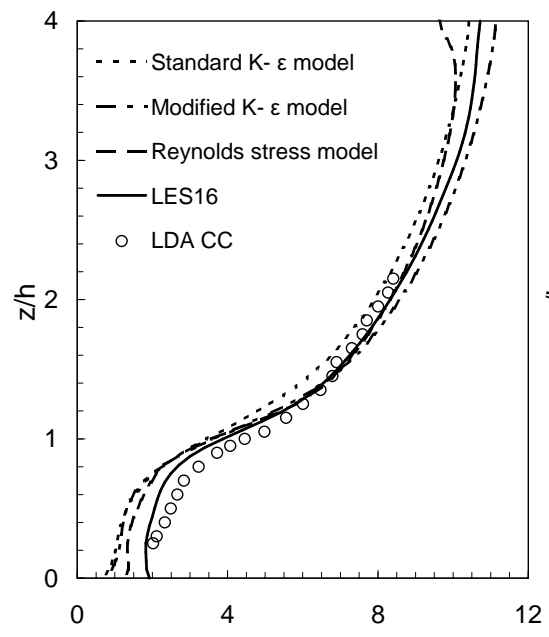

(a)

$\mathrm{U} / \mathrm{u}_{*}$

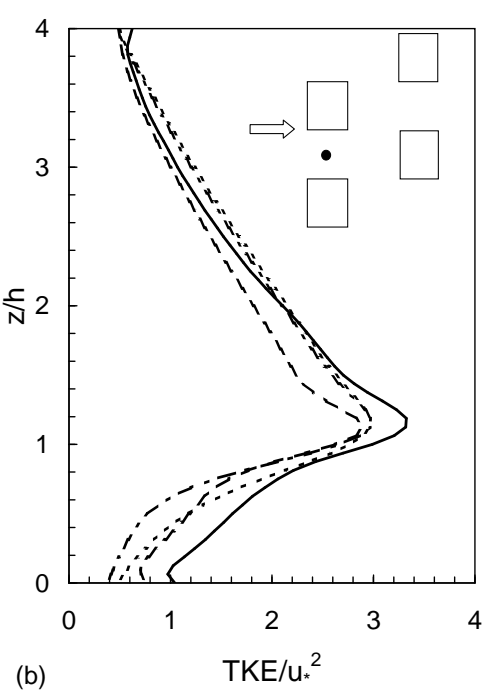

Figure 13. Vertical profiles of streamwise velocity and turbulence kinetic energy.

Figure 14 shows a comparison of mean velocity vectors in the $x-y$ plane at $z / h=0.5$ given by RANS and LES16. LES generates a large separation bubble on the lateral sides of cube, whereas RSM generates a weaker separation bubble. A separation bubble is hardly noticeable with the SKE and MKE models. Nevertheless it seems that there is a slight improvement in the predicted mean velocity at the front corners with the MKE compared with SKE model.

Figure 15 shows vertical profiles of Reynolds shear stress behind and in front of the cube from the RANS models and LES. In Figure 15a it is seen that MKE and RSM perform better that SKE in simulating the strong shear layer at the height of cube. All of the RANS models failed to simulate the zero shear stress at $0.3 h$ found by LDA measurement and DNS computations (see Figure 15b and Figure 7c). Among the three RANS models, SKE arguably performs the worst.

Turning back to Figure 11, profiles obtained from the three RANS models have a much higher peak at approximately $0.9 h$ compared with that suggested by LES, DNS and experimental data. It was also noted (but cannot be deduced from Figure 11) that all the three RANS models underestimate the sectional drag force below $z=0.8 h$, which is consistent with the excessive peak at approximately $z=0.9 h$ because the identical total driving force was imposed in all cases. This is also 


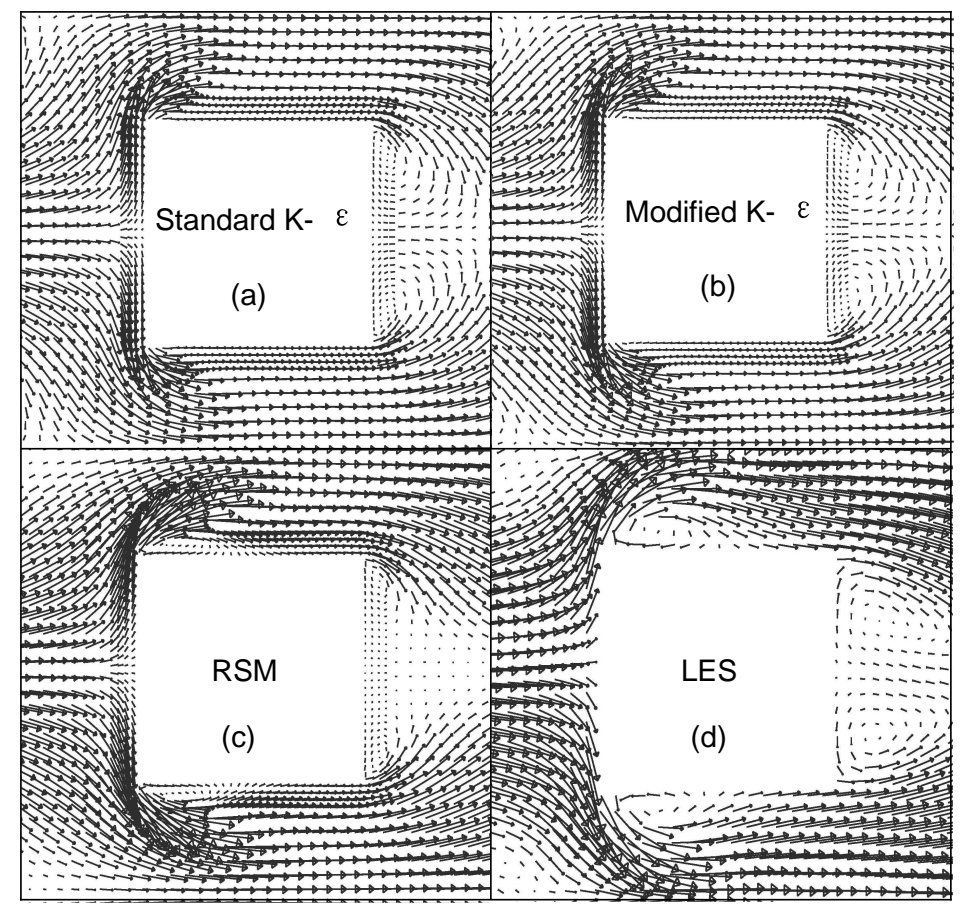

Figure 14. Mean velocity vectors in the $\mathrm{x}-\mathrm{y}$ plane at $z / h=0.5$. LES, medium mesh.

consistent with data in Figure 13a, which show that all three RANS models underestimate the mean streamwise velocity within the canopy. Not surprisingly, RSM yields the most accurate pressure profiles among the three RANS models.

\section{Final Discussion and Conclusions}

Overall, the comparisons between a fully-resolved DNS computation of flow at $R e=5 \times 10^{3}$ over a staggered array of cubes (Coceal et al., 2006) and corresponding LES16 computations are very satisfactory. The $R e$ is low enough for the LES32 to be 'almost DNS', but flows at $R e=5 \times 10^{4}$ and $R e=5 \times 10^{6}$, with coarse or fine grids, generated almost identical non-dimensional statistics compared with those at $R e=5 \times 10^{3}$, even though the high-frequency end of the spectral range was not well captured in some cases. This suggests that Reynolds number dependency (if it does exists) is very weak for such flows, except no doubt very close to solid walls. Turbulence generated by urban-like 

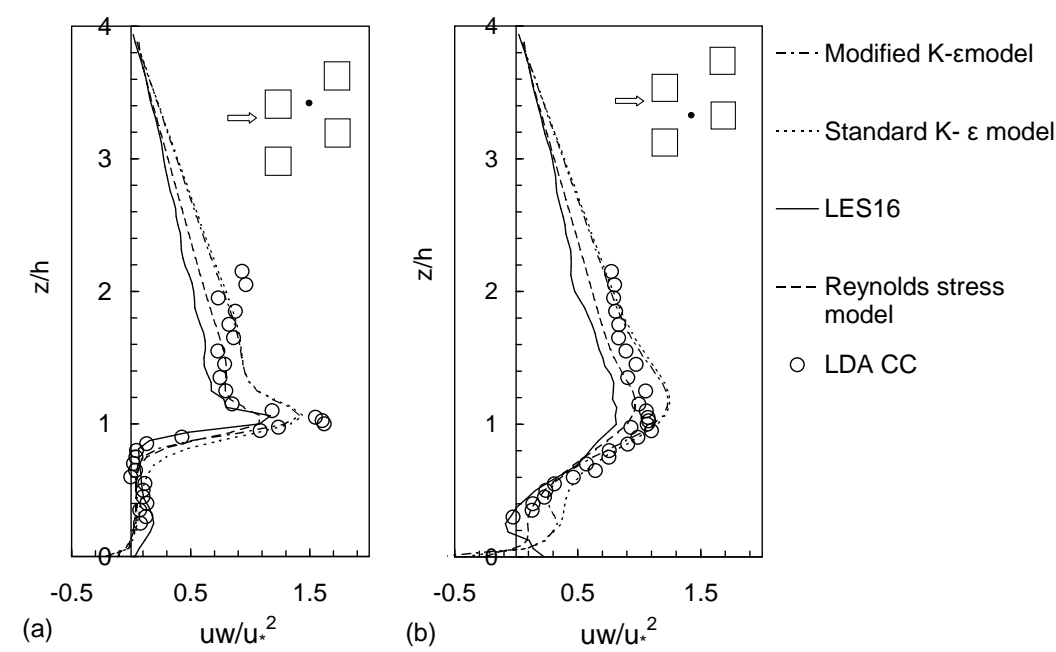

Figure 15. Vertical profiles of Reynolds shear stress behind and in front of the cube.

obstacles (with sharp edges) is large-scale dominated and hardly at all dependent on the much-smaller-scale viscous dominated processes on the body surfaces, so even at a low Reynolds number a relatively wide inertial sub-range exists in the near-surface layer (as seen in the measurements of Meinders \& Hanjalić 1999 and Castro et al.2005). These flows are thus much less Reynolds number dependent than, for example, flows over smooth walls or even around isolated bodies (Shah, 1998; Hoxey et al., 1998).

A wide inertial sub-range in flows over urban-like obstacles may also suggest that turbulence reaches a quasi-isotropic state at relatively lower frequency than non-vortex-shedding flows at similar Reynolds numbers, which is another reason why a simple SGS model can give reasonable results. Whilst improvement of the SGS model, via more expensive dynamic models for example, may enhance the simulation in the close vicinity of the solid walls at high Reynolds number and, likewise, improvements in the wall model itself, may increase accuracy near the element walls, it is much more important to use grids which can resolve the major features of the separated shear layers. The influence of the small-scale motions, increasingly inadequate as $R e$ rises, is much lower in these flows than it is in smooth-wall equivalents. This is all greatly beneficial for the numerical simulation of the coupling between weather scale flows and street scale flows. Our major conclusion is that LES may be reliably able to simulate turbulent flow over urban areas 
at realistic Reynolds numbers, with what (in more 'classical' flows) would normally be thought of as inadequate grids. It is suggested that medium sized meshes on the body scale (e.g. 15-20 grid points at least over a typical body dimension) are sufficient for the simulation of a real urban area, at least as far as obtaining the total drag force or the large-scale flow dynamics.

The results obtained from steady RANS models are generally comparable with those from LES above the canopy. However, it was found that within the canopy the results obtained from steady RANS models are much less satisfactory, compared with LES, which suggests that the inherent unsteadiness there plays an important role in such flows. It should also be stressed that a pure unsteady RANS method could not successfully be applied for such flows in view of the lack both of any significant scale separation and of any significant periodicity. Although the improvement from LES for such flows is at the cost of much more CPU time, e.g. at least an order of magnitude more than RANS, with the rapid advances in computing hardware and software such an expense is increasingly becoming affordable even for industrial-scale problems. To emphasise the point, the present numerical simulations with three million cells were performed on a local Linux PC cluster system, using up to $16 \mathrm{CPUs}$ and required about three weeks for adequate averaging.

Finally, it is worth pointing out that compared with a constant driving force, a time-varying external force can make a dramatic difference to turbulent flows. In their study of channel flow with a roughened (rippled) wall, for example, Chang \& Scotti (2004) found that the effect of an oscillation in the imposed pressure gradient was to increase the mean drag noticeably. Nevertheless, so far, statistically unsteady turbulent flows driven by time-varying external forces have received little attention, compared with the statistically steady ones (Dong et al., 2004). Our longer-term objective is to develop tools for implementing unsteady spatial boundary conditions derived from the output of much larger-scale computations, e.g. the UK Met Office's Unified Model, with an LES code for computing the street-scale flow. To maintain accuracy this requires the use of polyhedral meshes; further work on these topics will be reported in due course.

\section{Acknowledgment}

This project was supported by NCAS, NERC under the Urban Meteorology theme of the Universities Weather Research Network (UWERN, Grant No. DST/26/39). Numerical simulations were performed on the IRIDIS computational system, Information System Services (ISS), Uni- 
versity of Southampton and we are grateful for help from ISS staff. The CFD code providers were also very helpful in ensuring appropriate implementations of their codes on the ISS system and in discussing various aspects of the results presented here; we are very grateful to them all.

\section{References}

Addad Y., Laurence D., Talotte C., Jacob M.C. (2003) Large eddy simulation of a forward-backward facing step for acoustic source identification.

Andrén A., Brown A., Mason P.J., Graf J., Schumann U., Moeng C.-H and Nieuwstadt F.T.M. (1994) A neutrally stratified boundary-layer: a comparison of four large-eddy simulation computer codes. Direct and Large-Eddy Simulation I: Selected Paper from the First ERCOFTAC Workshop on Direct and LargeEddy Simulation, Eds: Voke P.R., Kleiser L. and Chollet J.-P., Kluwer Academic Publishers.

Apsley D.(1995) Numerical modelling of neutrally and stably stratified flow and dispersion in complex terrain, PhD thesis, University of Surrey, UK.

Britter R.E. and Hanna S.R. (2003) Flow and dispersion in urban areas, Annu. Rev. Fluid Mech. 35 469-496.

Castro I.P., Cowan I.R. and Robins A.G. (1999) Simulation of flow and dispersion around buildings, J. Aero. Eng., Oct. 145-160.

Castro I.P. (2003) CFD for external aerodynamics in the built environment, The QNET-CFD network Newsletter, 2:2. 229-259.

Castro I.P., Cheng H. and Reynolds R.(2005) Turbulence over urban-type roughness:decutions from with tunnel measurements, Bound.-layer Meteoro., 118: 109-131.

Chang Y.S. and Scotti A. (2004) Modeling unsteady turbulent flows over ripples: Reynolds-averaged Navier-Stokes equation (RANS) versus large-eddy simulation (LES), J. Geohys. Res. 109.

Cheng H. and Castro I.P. (2002a) Near wall flow over urban-like roughness, Boundary-Layer Meteorol. 104 229-259.

Cheng Y., Lien F.S., Yee E. and Sinclair R.(2003) A comarison of large eddy simulation with a standard $k-\epsilon$ Reynolds-averaged Navier-Stokes model for the prediction of a fully developed turbulent flow over a matrix of cubes, J. Wind Eng. Indus. Aerodyn. 91 1301-1328.

Coceal O., Thomas T.G., Castro I.P. and Belcher S.E. (2006) Mean flow and turbulence statistics over groups of urban-like cubical obstacles, Boundary Layer Meteorol., in press.

Daly B. J. and Harlow F. H. (1970) Transport Equations in Turbulence, Phys. Fluids, 13, 2634-2649.

Dong Y.H. and Lu X.Y. (2004) Large eddy simulation of a thermally stratified turbulent channel flow with temperature oscillation on the wall, Int. J. Heat Mass Transfer 47 2109-2122.

Dittrich A., Nestmann F., Ergenzinger P. (1996) Ratio of lift and shear forces over rough surfaces. In: Coherent flow structures in open channels. Ed.: Ashworth, P.J., et al.Wiley, New York.

Fackrell J.E. and Robins A.G.(1982) Concentration fluctuations and fluxes in plumes from point sources in a turbulent boundary layer, J. Fluid Mech., 117, 1-26. 
Finnigan J. (2000) Turbulence in plant canopy, Annu. Rev. Fluid Mech., 32, 519-571.

Gibson M. M. and Launder B. E. (1978) Ground Effects on Pressure Fluctuations in the Atmospheric Boundary Layer. J. Fluid Mech., 86,491-511.

Hanna S.R., Tehranian S., Carissimo B., Macdonald R. W. \& Lohner R. (2002) Comparisons of model simulations with observations of mean flow and turbulence within simple obstacle arrays. Atmos. Environ. 36 5067-5079.

Hoxey R.P., Reynolds A.M., Richardson G.M., Robertson A.P. and Short J.L. (1998) Observations of Reynolds number sensitivity in the separated flow region on a bluff body, J. Wind Eng. Ind. Aerodyn. 73 231-249.

Kanda M., Moriwaki R. \& Kasamatsu F. (2004) Large-eddy simulation of turbulent organized structures within and above explicitly resolved cube arrays. BoundaryLayer Meteorol. 112 343-368.

Kim S.-E. and Choudhury D. (1995) A Near-Wall Treatment Using Wall Functions Sensitized to Pressure Gradient. ASME FED Vol. 217, Separated and Complex Flows.

Leschziner M.A. \& Rodi W. (1981) Calculation of annular and twin jets using various discretisation schemes and turbulence model variations. J. Fluids Engrg. 103 352-360.

Mason P.J. (1989) Large-eddy simulation of the convective boundary layer, J. Atmos. Sci.46 1492-1516.

Meinders E.R. \& Hanjalić K.(1999) Vortex structure and heat transfer in turbulent flow over a wall-mounted matrix of cubes, Int. J. Heat Fluid Flow 20 255-267.

Moeng C.-H (1984) A large-eddy simulation model for the study of planetary boundary-layer turbulence, J. Atmos. Sci.41 2052-2062.

Nieuwstadt F.T.M and Brost R.A. (1986) The decay of convective turbulence, J. Atmos. Sci.43 532-546.

Shah K. B. (1998) Large eddy simulations of flow past a cubic obstacle. Dissertation, Stanford University.

Schmidt H. and Schumann U.(1989) Coherent structure of the convective boundary layer derived from large-eddy simulation, J. Fluid Mech. 200 511-562.

Stoesser T., Mathey F., Frohlich J. and Rodi W. (2003) LES of flow over multiple cubes. ERCOFTAC Bulletin No. 56.

Sagaut, P., (2001) Large Eddy Simulation for Incompressible Flows. Springer-Verlag.

Stull R. B.: 1993, An Introduction to Boundary Layer Meteorology, Kluwer Academic, the Netherlands, $366 \mathrm{pp}$.

Werner H. and Wengle H. (1991) Large-eddy simulation of turbulent flow over and around a cube in a plate channel. 8th Symposium on Turbulent Shear Flows, Munich, Germany.

Williams G.P. (1969) Numerical investigation of the three-dimensional Navier-Stokes equations for incompressible flow, J. Fluid Mech. 37, 727-750.

Xie Z., Voke P.R., Hayden P. and Robins G.A. (2004) Large-eddy simulation of turbulent flow over a rough surface. Boundary-Layer Meteorol. 111 417-440.

Xie Z., Hayden P., Voke P.R. and Robins A.G.,(2004) Large-eddy simulation of dispersion: comparison between elevated source and ground level source. $J$. Turbulence. 5(31), 1-16.

Address for Offprints:

KLUWER ACADEMIC PUBLISHERS PrePress Department,

P.O. Box 17, 3300 AA Dordrecht, The Netherlands 
e-mail: TEXHELP@WKAP.NL

Fax: +31 786392500 